\section{Unraveling the effect of silent, intronic and missense mutations on VWF splicing: contribution of next generation sequencing in the study of mRNA} Teresa Fidalgo, ${ }^{4}$ Patricia Martinho, ${ }^{4}$ María Fernanda López-Fernández, ${ }^{3}$ Ángela Rodríguez-Trillo, ${ }^{3}$ Esther Lourés, ${ }^{3}$ Rafael Parra, ${ }^{1,2}$ Carme Altisent, ${ }^{2}$ Ana Rosa Cid, ${ }^{5}$ Santiago Bonanad, ${ }^{5}$ Noelia Cabrera, ${ }^{5}$ Andrés Moret, ${ }^{5}$ María Eva Mingot-Castellano, ${ }^{6}$ Nira Navarro, ${ }^{7}$ Rocío Pérez-Montes, ${ }^{8}$ Sally Marcellin, ${ }^{9}$ Ana Moreto, ${ }^{10}$ Sonia Herrero, ${ }^{11}$ Inmaculada Soto, ${ }^{12}$ Núria Fernández-Mosteirín, ${ }^{13}$ Víctor Jiménez-Yuste, ${ }^{14}$ Nieves Alonso, ${ }^{15}$ Aurora de Andrés-Jacob, ${ }^{16}$ Emilia Fontanes, ${ }^{17}$ Rosa Campos, ${ }^{18}$ María José Paloma, ${ }^{19}$ Nuria Bermejo, ${ }^{20}$ Ruben Berrueco, ${ }^{21}$ José Mateo, ${ }^{22}$ Karmele Arribalzaga, ${ }^{23}$ Pascual Marco,${ }^{24}$ Ángeles Palomo, ${ }^{25}$ Nerea Castro Quismondo, ${ }^{26}$ Belén Iñigo, ${ }^{27}$ María del Mar Nieto, ${ }^{28}$ Rosa Vidal, ${ }^{29}$ María Paz Martínez, ${ }^{30}$ Reyes Aguinaco, ${ }^{31}$ Jesús María Tenorio, ${ }^{33}$ María Ferreiro, ${ }^{33}$ Javier García-Frade, ${ }^{34}$ Ana María Rodríguez-Huerta, ${ }^{35}$ Jorge Cuesta, ${ }^{36}$ Ramón Rodríguez-González, ${ }^{37}$ Faustino García-Candel, ${ }^{38}$ Manuela Dobón, ${ }^{39}$ Carlos Aguilar, ${ }^{40}$ Francisco Vidal ${ }^{1,2,41}$ and Irene Corrales ${ }^{1,2}$

${ }^{1}$ Banc de Sang i Teixits, Barcelona, Spain; ${ }^{2}$ Institut de Recerca Vall d'Hebron Universitat Autònoma de Barcelona (VHIR-UAB), Spain; ${ }^{3}$ Complexo Hospitalario Universitario A Coruña, INIBIC, Spain; ${ }^{4}$ Centro Hospitalar e Universitário de Coimbra, Portugal; ${ }^{5} \mathrm{Hospital}$ Universitario y Politécnico La Fe, Valencia, Spain; ${ }^{6} \mathrm{Hospital}$ Regional Universitario de Málaga, Spain; ${ }^{7}$ Hospital Universitario Dr. Negrín, Las Palmas de Gran Canaria, Spain; ${ }^{8}$ Hospital Universitario Marqués de Valdecilla, Santander, Spain; ${ }^{9}$ Salud Castilla y León, Segovia, Spain; ${ }^{10}$ Hospital Universitario Cruces, Barakaldo, Spain; ${ }^{11}$ Hospital Universitario de Guadalajara, Spain; ${ }^{12}$ Hospital Universitario Central de Asturias, Oviedo, Spain; ${ }^{13}$ Hospital Universitario Miguel Servet, Zaragoza, Spain; ${ }^{14}$ Hospital Universitario La Paz, Madrid, Spain; ${ }^{15} \mathrm{Hospital}$ Infanta Cristina, Badajoz, Spain; ${ }^{16}$ Complexo Hospitalario Universitario Santiago de Compostela, Spain; ${ }^{17}$ Hospital Universitario Lucus Augusti, Lugo, Spain; ${ }^{18} \mathrm{Hospital}$ Jerez de la Frontera, Cádiz, Spain; ${ }^{19} \mathrm{Hospital}$ Virgen del Camino, Pamplona, Spain; ${ }^{20} \mathrm{Hospital}$ San Pedro de Alcántara, Cáceres, Spain; ${ }^{21} \mathrm{Hospital}$ Sant Joan de Deu, Barcelona, Spain; ${ }^{22} \mathrm{Hospital}$ Sta Creu i St Pau, Barcelona, Spain; ${ }^{23}$ Hospital Universitario Fundación de Alcorcón, Madrid, Spain; ${ }^{24} \mathrm{Hospital}$ General de Alicante, Spain; ${ }^{25} \mathrm{Hospital}$ Regional Universitario Carlos Haya, Málaga, Spain; ${ }^{26}$ Hospital Universitario 12 de Octubre, Madrid, Spain; ${ }^{27} \mathrm{Hospital}$ Clínico San Carlos, Madrid, Spain; ${ }^{28}$ Complejo Hospitalario de Jaén, Spain; ${ }^{29}$ Fundación Jiménez Díaz, Madrid, Spain; ${ }^{30} \mathrm{Hospital}$ Nuestra Sra. de Sonsoles de Ávila, Spain; ${ }^{32} \mathrm{Hospital}$ Joan XXIII, Tarragona, Spain; ${ }^{32}$ Hospital Ramón y Cajal, Madrid, Spain; ${ }^{33} \mathrm{Hospital}$ Montecelo, Pontevedra, Spain; ${ }^{34}$ Hospital Río Hortega, Valladolid, Spain; ${ }^{35}$ Hospital Gregorio

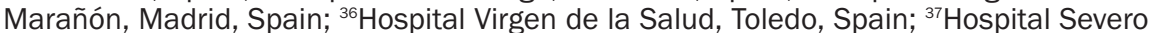
Ochoa, Madrid, Spain; ${ }^{38} \mathrm{Hospital}$ Universitario Virgen Arrixaca, Murcia, Spain; ${ }^{39} \mathrm{Hospital}$ Lozano Blesa, Zaragoza, Spain; ${ }^{40} \mathrm{Hospital}$ Santa Bárbara, Soria, Spain and ${ }^{41} \mathrm{CIBER}$ de Enfermedades Cardiovasculares, Madrid, Spain

\section{ABSTRACT}

T arge studies in von Willebrand disease patients, including Spanish and Portuguese registries, led to the identification of $>250$ different mutations. It is a challenge to determine the pathogenic effect of potential splice site mutations on VWF mRNA. This study aimed to elucidate the true effects of 18 mutations on VWF mRNA processing, investigate the contribution of next-generation sequencing to in vivo mRNA study in von Willebrand disease, and compare the findings with in silico prediction. RNA extracted from patient platelets and leukocytes was amplified by RT-PCR and sequenced using Sanger and next generation sequencing techniques. Eight mutations affected VWF splicing: c. $1533+1 \mathrm{G}>\mathrm{A}$, c. $5664+2 \mathrm{~T}>\mathrm{C}$ and c.546G $>\mathrm{A}(\mathrm{p} .=)$ prompted exon skipping; c.3223-7_3236dup and c.7082-2A $>\mathrm{G}$ resulted in activation of cryptic sites; c.3379+1G>A and c.7437G $>$ A) demonstrated both molecular pathogenic mechanisms simultaneously; and the p.Cys370Tyr missense mutation generated two aberrant transcripts. Of note, the complete
Ferrata Storti Foundation

\author{
Haematologica 2019 \\ Volume 104(1):587-598
}

\section{Correspondence:}

IRENE CORRALES

icorrales@bst.cat/fvidal@bst.cat

Received: August 3, 2018.

Accepted: October 19, 2018.

Pre-published: October 25, 2018.

doi:10.3324/haematol.2018.203166

Check the online version for the most updated information on this article, online supplements, and information on authorship \& disclosures: www.haematologica.org/content/104/3/587

\section{(C)2019 Ferrata Storti Foundation}

Material published in Haematologica is covered by copyright. All rights are reserved to the Ferrata Storti Foundation. Use of published material is allowed under the following terms and conditions:

https://creativecommons.org/licenses/by-nc/4.0/legalcode. Copies of published material are allowed for personal or internal use. Sharing published material for non-commercial purposes is subject to the following conditions:

https://creativecommons.org/licenses/by-nc/4.0/legalcode, sect. 3. Reproducing and sharing published material for commercial purposes is not allowed without permission in writing from the publisher. 
effect of three mutations was provided by next generation sequencing alone because of low expression of the aberrant transcripts. In the remaining 10 mutations, no effect was elucidated in the experiments. However, the differential findings obtained in platelets and leukocytes provided substantial evidence that four of these would have an effect on VWF levels. In this first report using next generation sequencing technology to unravel the effects of VWF mutations on splicing, the technique yielded valuable information. Our data bring to light the importance of studying the effect of synonymous and missense mutations on VWF splicing to improve the current knowledge of the molecular mechanisms behind von Willebrand disease. clinicaltrials.gov identifier:02869074.

\section{Introduction}

Von Willebrand disease (VWD), the most common congenital bleeding disorder, is caused by a genetic defect in the von Willebrand factor gene (VWF). ${ }^{1}$ VWF mutational analysis can be valuable for diagnosing and investigating the molecular etiology of VWD, as was seen in the Spanish (PCM-EVW-ES project) and Portuguese cohort of VWD patients..$^{2-4}$ One interesting challenge in this condition is to elucidate the pathogenic mechanism of VWF mutations. In silico analysis is considered a suitable supporting tool to predict the pathogenicity of the variants identified. ${ }^{5,6}$ However, functional studies remain essential to unequivocally determine their deleterious effect.

Functional studies can be performed by analyzing the potential effect of splice site mutations (PSSM) in RNA. In addition to splice site consensus sequence mutations, deep intronic, missense, and synonymous mutations can also disturb splicing. Along this line, $25 \%$ of synonymous mutations positioned at exon-intron boundaries result in altered splicing, which, in itself, can cause disease, modify the severity of the disease phenotype, or be linked with disease susceptibility. ${ }^{8}$ In VWF, heterozygotes for PSSM may be associated with mild forms of VWD type 1 or be phenotypically silent, but when two such mutations are found in different alleles, the phenotype is associated with VWD type $3 .^{9}$

Almost all related studies of splicing effects have examined peripheral blood platelets, as VWF is exclusively expressed in these cells and endothelial cells. ${ }^{10,11}$ Platelets are anucleated, but they contain small amounts of translationally active megakaryocytic mRNA. ${ }^{12,13}$ In contrast, the amount of mRNA obtained from leukocytes is higher and contains mRNA transcripts for genes that are not normally expressed in these cells, known as "ectopic transcripts". Their analysis has been used to investigate mutations in several inherited disorders, ${ }^{14,15}$ as they facilitate the study of mRNA of those genes expressed in hard to reach tissues. ${ }^{16}$ PSSM can affect mRNA reorganization and introduce premature termination codons (PTCs) into open reading frames, a common cause of genetic disorders. Most nonsense transcripts are recognized and degraded by nonsense-mediated mRNA decay (NMD), ${ }^{17}$ a degradation pathway to control synthesis of truncated proteins. ${ }^{18}$ The efficiency of NMD varies between cell types; hence, the use of RNA from platelets and leukocytes for in vivo study of VWF PSSM offers complementary results, particularly when NMD occurs in the allele carrying the mutation in platelets, as we reported.

Since its development, next-generation sequencing (NGS) has been increasingly used in molecular genetics to identify mutations causing disease. However, few groups have explored its potential for analyzing splicing variants following RT-PCR. ${ }^{19,20}$ In this new scenario, the procedure we previously described to analyze the effects of PSSM in $V W F^{7}$ has been optimized and adapted to an NGS-based technique to investigate its value in this field. Our main objective was to elucidate the true effects of 18 selected mutations (intronic, synonymous, delins, and missense) on mRNA processing and their genotype/phenotype correspondence by analysis of leukocytes and platelets from clinical samples. Finally, the in vivo effects of the mutations were compared with the in silico predictions.

\section{Methods}

\section{Patients}

We studied 15 patients diagnosed with different types of VWD, 5 from Complexo Hospitalario Universitario A Coruña, 8 from Hospital Universitari Vall d'Hebron (HUVH), and 2 from Centro Hospitalar e Universitário de Coimbra. Samples from 4 healthy individuals were used as controls. The study was performed according to the guidelines of the Declaration of Helsinki and was approved by the local Research Ethics Committee. All participants provided written informed consent.

\section{Splice site prediction software}

The predicted impact of potential splice site mutations was analyzed with NetGene $2^{21}$ and the splicing prediction module of Alamut Visual v.2.6.1 software (Interactive Biosoftware, Rouen, France), which integrates data from three methods: Splice Site Prediction by Neural Network (NNSplice), MaxEntScan, and Human Splicing Finder (HSF).

\section{Platelet and leukocyte separation and RNA isolation}

Leukocyte and platelet RNA from patients and controls was isolated from $10 \mathrm{~mL}$ of peripheral blood collected in EDTA tubes, as previously described.

\section{VWF mRNA amplification}

After RNA isolation, cDNA was synthesized using the HighCapacity cDNA Reverse Transcription Kit (Thermo Fisher Scientific, Waltham, MA, USA) according to the manufacturer's recommendations. The region including the mutation was amplified by Platinum Taq DNA Polymerase (Thermo Fisher Scientific) in leukocyte and platelet cDNA (Online Supplementary Methods and Table S1). PCR products were separated on 1\% agarose gel and visualized by SYBR Safe DNA Gel Stain (Thermo Fisher Scientific).

\section{Sanger sequencing and analysis}

PCR products were sequenced as previously described. ${ }^{22}$ However, multiple-band PCR products were previously agarose- 
Table 1. Laboratory and molecular data of VWD patients

\begin{tabular}{|c|c|c|c|c|c|c|c|c|c|}
\hline Patients code & WW & WWFAg & WWFRRO & FVIIII:C & NT change & AA change & Exon & Intron & Domain \\
\hline UMP01 & 3 carrier & 70 & 70.5 & 111 & c. $1533+1 G>A$ & - & - & 13 & intronic \\
\hline \multirow[t]{2}{*}{ UMP02 } & $1 \mathrm{H}$ & 36 & 49 & 101 & c. $3379+1 G>A *$ & - & - & 25 & intronic \\
\hline & & & & & c. $-2627 \mathrm{C}>\mathrm{T}$ * & - & - & - & upstream \\
\hline \multirow[t]{2}{*}{ UMP03 } & 1 & 26 & 21.2 & 64.8 & c.5664+2T $>C$ * & - & - & 33 & intronic \\
\hline & & & & & c. $7220 \mathrm{~T}>\mathrm{C}$ * & p.Leu2407Pro & 42 & - & $\mathrm{C} 2$ \\
\hline UMP04 & 1 & 50 & 52 & 47 & c. $7081+6 G>T$ & - & - & 41 & intronic \\
\hline UMP05 & 1 & 27 & 32 & 72 & c. $7730-56 \mathrm{C}>\mathrm{T}$ & - & - & 45 & intronic \\
\hline \multirow[t]{2}{*}{ UMP06 } & $2 \mathrm{~A} / 2 \mathrm{M}$ & 12 & 4 & 24 & c. $4120 \mathrm{C}>\mathrm{T}^{*}$ & p.Arg1374Cys & 28 & & $\mathrm{Al}$ \\
\hline & & & & & c. $7730-4 C>G *$ & - & - & 45 & intronic \\
\hline UMP07 & 1 & 37 & 28 & 60 & c.8254-5T>G & - & - & 51 & intronic \\
\hline \multirow[t]{2}{*}{ UMP08 } & 1 & 42.4 & 41.7 & 73.4 & c.546G $>\mathrm{A}^{\dagger}$ & p.Ser182 & 6 & - & D1 \\
\hline & & & & & c. $4866 \mathrm{C}>\mathrm{T}^{\dagger}$ & p.Asp1622 & 28 & - & Al-A3 \\
\hline UMP09: & 1 & 51 & 49.8 & 71 & c.3291C $>\mathrm{T}$ & p.Cys1097 & 25 & - & D3 \\
\hline UMP10 & 3 carrier & 46.4 & 45 & 105 & c. $1109 \mathrm{G}>\mathrm{A}$ & p.Cys370Tyr & 9 & - & D1 \\
\hline \multirow[t]{2}{*}{ UMP11 } & 1 & 31 & 24 & 57 & c.3223-7_3236dup & p.Pro1079_Tyr1080ins & 25 & - & D3 \\
\hline & & & & & & LeuGlnValAspProGluPro & & & \\
\hline \multirow[t]{2}{*}{ UMP12 } & 3 & 3.7 & 5 & 6.7 & c. $449 \mathrm{~T}>\mathrm{C}^{\dagger}$ & p.Leu150Pro & 5 & - & D1 \\
\hline & & & & & c. $7082-2 A>G^{\dagger}$ & - & - & 41 & intronic \\
\hline \multirow[t]{3}{*}{ UMP13 } & $2 \mathrm{~A}$ & 117 & 6.5 & 90 & c.3426T $>\mathrm{C}^{\S}$ & p.Cys1142 & 26 & - & D3 \\
\hline & & & & & c.3485_3486delins'TG ${ }^{\S}$ & p.Pro1162Leu & 26 & - & D3 \\
\hline & & & & & c. $8318 \mathrm{G}>\mathrm{C}^{\dagger}$ & p.Cys2773Ser & 52 & - & CK \\
\hline \multirow[t]{2}{*}{ UMP14 } & 1 & 6 & 6 & 4,5 & c.6699_6702dup ${ }^{\dagger}$ & p.Cys2235ArgfsTer8 & 38 & - & D4 \\
\hline & & & & & c. $7437 \mathrm{G}>\mathrm{A}^{\dagger}$ & p.Ser2479 & 43 & - & C3 \\
\hline \multirow[t]{3}{*}{ UMP15" } & 3 & 2 & $<1$ & 5 & c. $546 \mathrm{G}>\mathrm{A}^{\S}$ & p.Ser182 & 6 & - & D1 \\
\hline & & & & & c. $7082-2 A>G^{\S}$ & - & - & 41 & intronic \\
\hline & & & & & c. $8155+3 \mathrm{G}>\mathrm{C}^{\dagger}$ & - & - & 50 & intronic \\
\hline
\end{tabular}

All mutations were identified in heterozygous state. In bold, mutations selected to study their effect on $V W F$ mRNA. The subtype $1 \mathrm{H}$ (historical) refers to patients previously diagnosed as type 1 VWD that, at the time of enrollment at the PCM-EVW-ES project, show a slight decrease or even a normal VWF plasma levels. AA:amino acid; NT: nucleotide; VWD: von Willebrand disease. *The allelic phase (cis/trans) of mutations could not be determined since no informative relatives are available. 'Mutations in trans. ${ }^{*}$ Patient with an additional mutation in the F8 gene. ${ }^{5}$ Mutations in cis. "Patient previously studied at mRNA level by Corrales et al.

purified using the MiniElute Gel Extraction kit (Qiagen). The sequences obtained were assembled and aligned against the consensus wild-type (WT) VWF mRNA sequence (GenBank NM_000552) using SeqScape v2.7 software (Thermo Fisher Scientific).

\section{Next-generation sequencing and analysis}

PCR amplicons obtained per patient were equimolarly mixed in a single tube in a total amount of $250 \mathrm{ng}$. Subsequently, the libraries were fragmented and the adapter and barcodes were ligated using the NGSgo protocol (GenDX, Utrecht, Netherlands) following the manufacturer's recommendations. Resulting libraries were combined and sequenced on a MiSeq platform (Illumina, San Diego, CA).

After sequencing, barcoded sequences were demultiplexed and analyzed individually. The paired sequence files (fastq format) were used as input for analysis with the CLC Genomic Workbench v.11 software (Qiagen, Aarhus, Denmark) (Online Supplementary Methods and Figure S1-S2).

\section{Results}

An in-depth study was performed in PCM-EVW-ES, ${ }^{3}$ the Portuguese cohort, ${ }^{4}$ and HUVH patients to select previous- ly undescribed VWF mutations and mutations with an unknown or controversial pathogenic mechanism. Eighteen mutations (15 patients) were selected: 8 intronic (4 in canonical, GT and AG, splice site sequences), 5 synonymous, 2 missense, and 3 delins. The patients' phenotypic and molecular data are summarized in Table 1 and Online Supplementary Table S2. Total mRNA was obtained from platelets and leukocytes of all patients, with the exception of patients UMP08 and UMP14, in whom platelet RNA isolation failed due to blood lysis. All mutations were analyzed by both Sanger sequencing and NGS, and results were compared to the predictions generated by in silico analysis (Online Supplementary Table S3).

\section{Mutations in canonical splice site sequences}

The c.1533+1G>A mutation (intron 13) was identified in a type 3 VWD carrier (UMP01). The exon 11-15 region was analyzed with a specific primer pair to avoid amplification of a prevalent alternative-splicing product (skipping of exons 14 and 15) in VWF from leukocytes. 'Whereas only the expected PCR product was observed in platelets, leukocyte amplification resulted in 4 PCR bands. Sanger analysis of leukocytes showed 2 mRNA aberrant transcripts: 1) lacking exon 13; and 2) lacking exon 13 and 14 (Online Supplementary Figure S3). By means of NGS, it was 
A

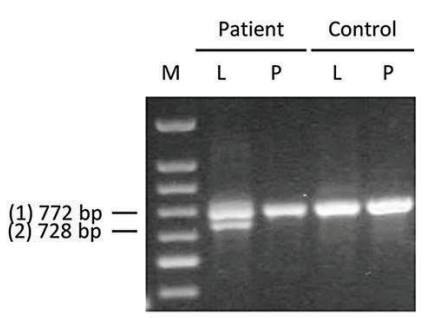

B

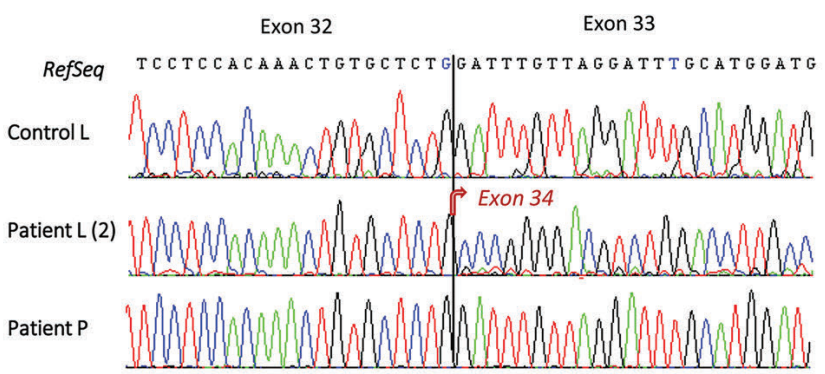

C

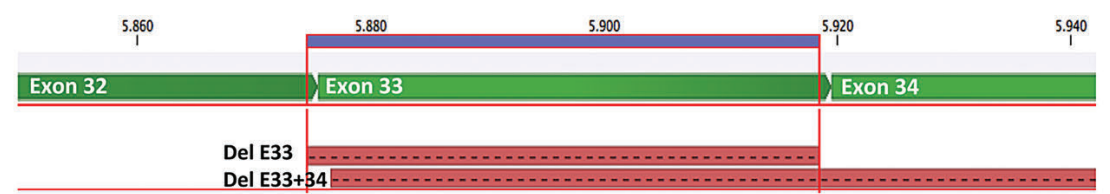

Output Indel and SV analysis

Coverage

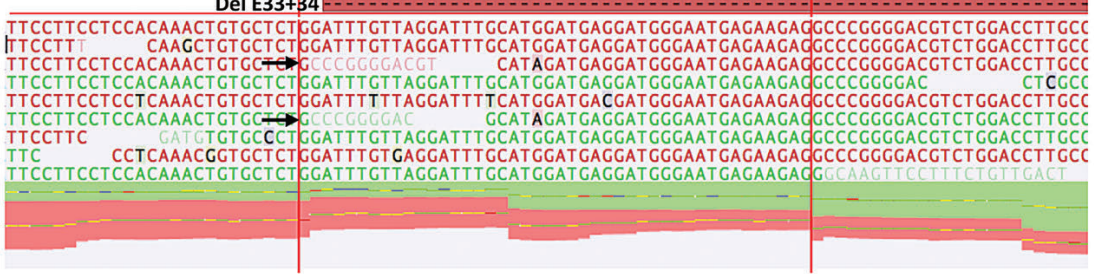

D

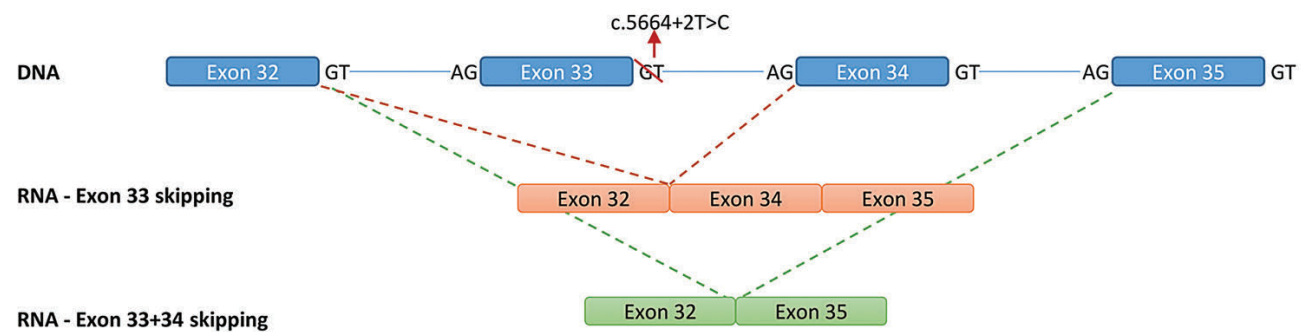

Figure 1. Analysis of the c.5664+2T>C mutation in patient UMP03. A) RT-PCR products amplified with primers located in exons 30 and 35 in leukocyte (L) and platelet (P) RNA, separated on 1\% agarose gel. B) Traditional Sanger sequencing of PCR products from patient leukocytes (L2) and platelets (P), and control leukocytes (L). Analysis of the band 2 from patient leukocyte on agarose gel demonstrates exon 33 skipping. In platelets, only the allele without c.5664+2T>C mutation could be amplified. C) NGS of PCR products from leukocytes showed exon 33 skipping, indicated by arrows depict aberrant transcripts. However, exon 33-34 skipping was also detected, but in a really low of transcripts. D) Schematic representation of the mutation in genomic DNA and its effect on the VWF mRNA sequence. M indicates a 100-bp DNA ladder.

determined that $19 \%$ were transcripts without exon 13 , $45 \%$ transcripts without exons 13 and 14 , and an additional aberrant transcript without exon 14 was detected in $8 \%$ transcripts (Online Supplementary Table S4). By both techniques, no effect was visible in platelets due to NMD.

The c.3379+1G>A mutation (intron 25) was identified in a type $1 \mathrm{H}$ VWD patient (UMP02). We designed two new primers to analyze the exon 22-26 region. Amplification of leukocyte cDNA yielded two bands, one with the expected size and a smaller band, whereas in platelets only the expected PCR product was observed (Online Supplementary Figure S4). Sanger analysis of leukocytes showed that c.3379+1G >A caused exon 25 skipping, leading to a frameshift at position 1075 and adding 88 aberrant amino acids before a PTC was encountered (p.Pro1075ValfsTer88). On NGS, however, two aberrant transcripts were detected: the major one, which lacked exon 25 , was detected in $43 \%$ of all reads, and the minor one, which resulted from activation of a cryptic donor splice site (DSS) 31 nucleotides upstream from the WTDSS, was found in $1.4 \%$ of reads (p.Pro1117ValfsTer88) (Table 2 and Online Supplementary Table S4). In platelets, however, the mutated allele had undergone $\mathrm{NMD}$, which precluded observation of aberrant transcripts.

The c.5664+2T>C mutation was identified in a type 1 VWD patient (UMP03), in trans with the p.Leu2407Pro mutation. To analyze the PSSM in intron 33, the exon 3035 region was amplified. The PCR product had the expected size in platelets, whereas an additional smaller one was observed in leukocytes (Figure 1). Sanger sequencing of the leukocyte PCR product confirmed exon 33 skipping (p.Gly1874AlafsTer32) (Table 2). NGS detected 2 aberrant 


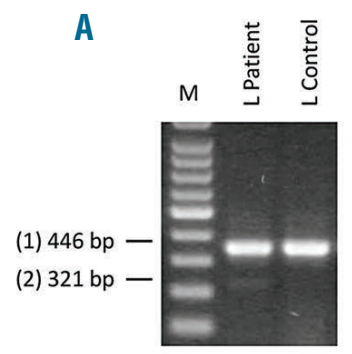

B
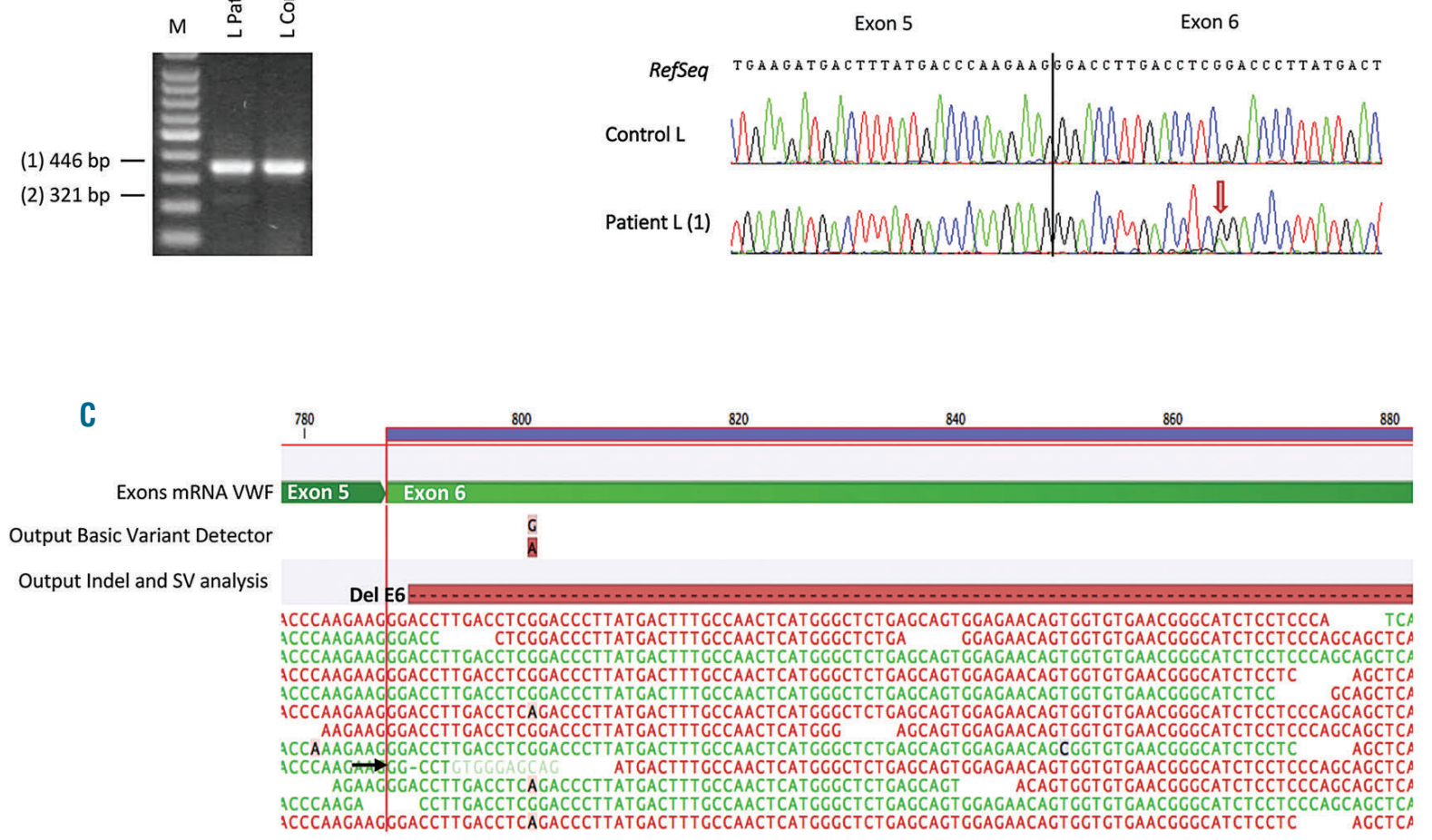

D

DNA

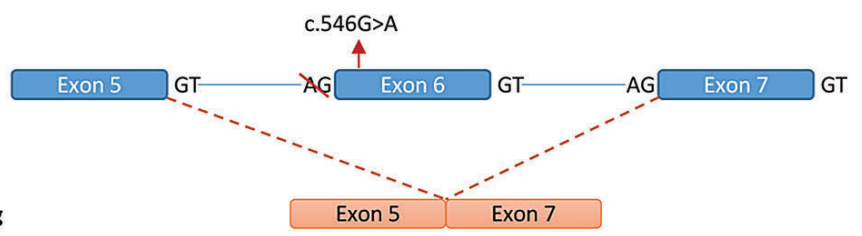

RNA - Exon 6 skipping

Exon 5 Exon 7

Figure 2. Analysis of the c.546G>A mutation, located at nucleotide 14 from the beginning of exon 6 , in patient UMP08. A) RT-PCR products amplified with primers located in exons 4 and 7 in leukocyte RNA (L), separated on 1\% agarose gel. B) Traditional Sanger sequencing of PCR product from patient L (1) showed the single nucleotide change. C) NGS of PCR products from leukocytes identified the single nucleotide variant, as well as exon 6 skipping. Arrows show aberrant transcripts. D) Schematic representation of the mutation in genomic DNA and its effect on the VWF mRNA sequence. M indicates a 100-bp DNA ladder.

transcripts: the major one (37\% of reads) showed exon 33 skipping, and the minor ( $2 \%$ of reads) showed exons 33 and 34 skipping. Moreover, analysis of the p.Leu2407Pro mutation confirmed that $\mathrm{NMD}$ of the allele carrying the c.5664+2T $>$ C mutation had occurred in platelets.

\section{Candidate intronic mutations}

The c.7081+6G $>$ T mutation, identified in a type 1 VWD patient (UMP04), generated a new GT dinucleotide in intron 41 (Online Supplementary Table S3). The exon 38-43 region showed the expected $\mathrm{PCR}$ product in both cell types (772 bp). To confirm these results, two informative SNPs (rs216321 in exon 20 and rs216902 in exon 35) were genotyped. Both were in heterozygous state, indicating that the allele carrying the mutation was unaffected by NMD.

The c.7730-56C>T mutation (intron 45) was identified in a type 1 VWD patient (UMP05). The exon 43-49 region, examined by Sanger and NGS, showed no visible effect on mRNA splicing. To confirm the presence of the allele carrying the intronic mutation, two informative SNPs were analyzed: rs216321 and rs1800380. Surprisingly, the sequence obtained in both cell types indicated that only one allele was expressed. To further explore these results, we tested whether intron 45 was retained within the mature mRNA (Online Supplementary Methods) and performed complete sequencing of VWF cDNA from leukocytes. Nonetheless, no changes were observed (data not shown).

The c.7730-4C > G mutation (intron 45) was identified in a type $2 \mathrm{~A} / 2 \mathrm{M}$ patient (UMP06), combined with the p.Arg1374Cys mutation. The HSF predicted activation of a cryptic intronic acceptor splice site (ASS), but in the exon 43-49 region, splicing was not affected by the mutation. To confirm the presence of the allele carrying c.7730$4 \mathrm{C}>\mathrm{G}$, the p.Arg1374Cys mutation was analyzed, and both nucleotides were seen in platelets and leukocytes, suggesting expression of both alleles. We then performed the same experiment as was done in patient UMP05 to test intron 45 retention, but no changes in VWF cDNA were observed (data not shown). 


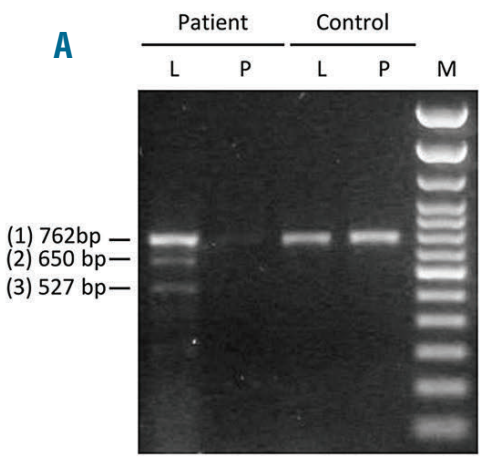

B
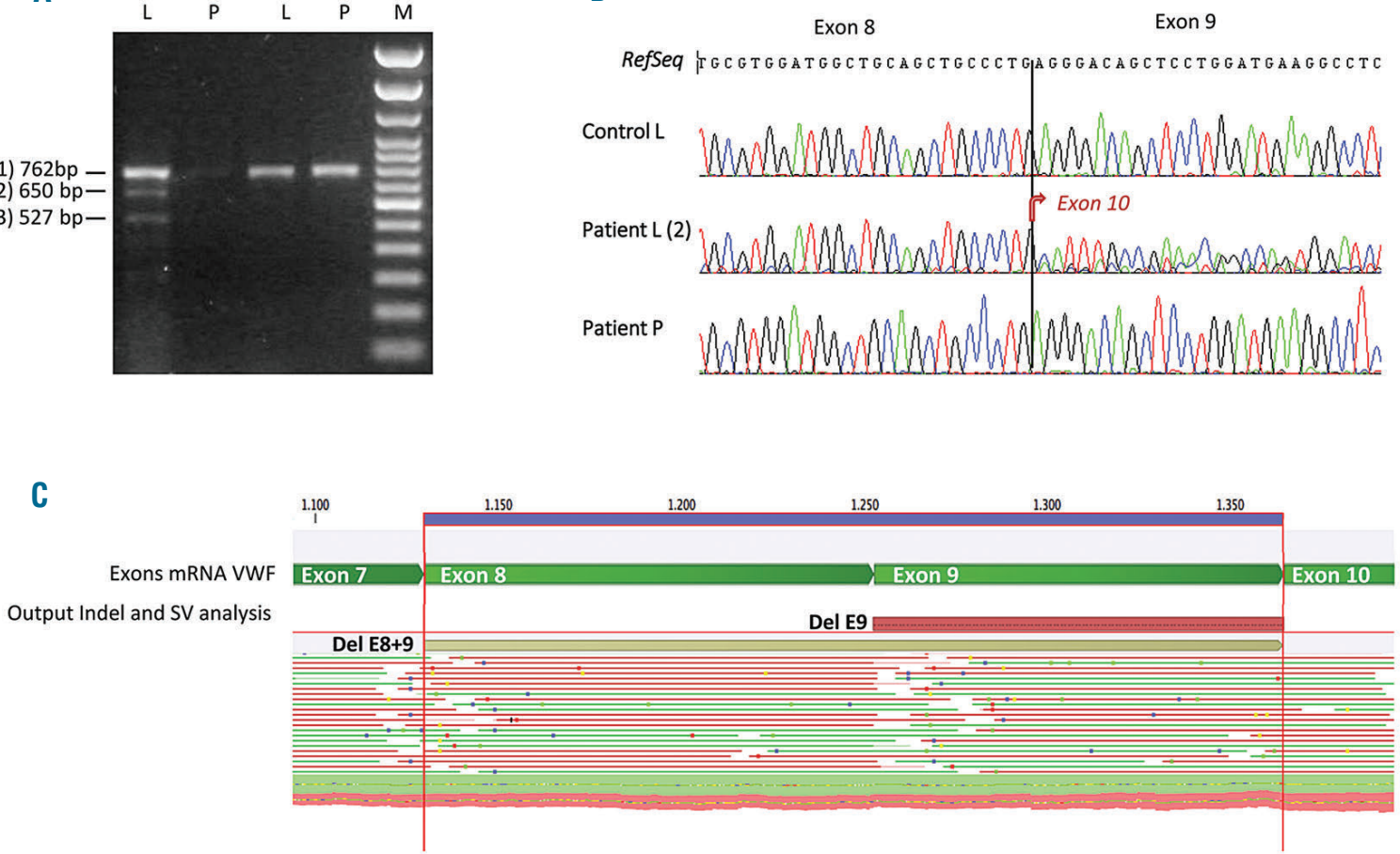

D

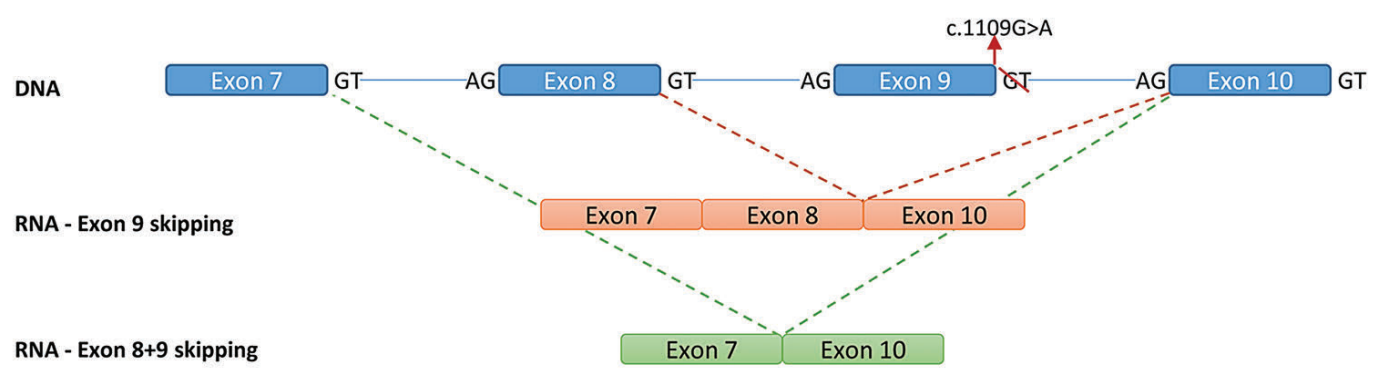

Figure 3. Analysis of the c.1109G>A (p.Cys370Tyr) mutation in patient UMP10, located in the last nucleotide of exon 9. A) RT-PCR products amplified with primers located in exons 6 and 12 in leukocyte $(L)$ and platelet $(P)$ RNA, separated on 1\% agarose gel. B) Traditional Sanger sequencing of PCR product from patient $L(2)$ showed exon 9 skipping. The $L(3)$ band could not be purified in the agarose gel due to low concentration, thus it was not analyzed by Sanger. In platelets, only the non-mutated allele could be amplified. C) NGS of PCR products from leukocytes identified transcripts lacking exon 9, as well as transcripts lacking exons 8 and 9. D) Schematic representation of the mutation in genomic DNA and its effect on the VWF mRNA sequence. M indicates a 100-bp DNA ladder.

The c.8254-5T>G mutation (intron 51) was found in a type 1 VWD patient (UMP07). The exon 49-52 region was amplified, and the expected 546-bp size was observed in both cell types. Sequence analysis by the two techniques revealed no changes in VWF cDNA. Interestingly, this patient's two children, heterozygous for the c.8254-5T>G mutation, had bleeding symptoms. Hence, we performed complete sequencing of VWF cDNA from leukocytes. However, no changes were identified.

\section{Synonymous mutations}

The mutations c.546G >A (exon 6) and c.4866C > T (exon 28) were identified in trans in a type 1 VWD patient (UMP08). These mutations were studied only in leukocyte RNA. To investigate c.546G $>$ A, the exon 4-7 region was amplified, which resulted in the expected band and a slightly diffuse, smaller band. Sanger sequencing detected the c.546G $>$ A change (Figure 2). However, NGS not only detected the nucleotide change, but also recognized a loss of 125 nucleotides corresponding to exon 6 (p.Thr179ProfsTer31) in small $2.3 \%$ of reads (Online Supplementary Table S4). The predicted impact of the c.546G >A mutation was discrepant and only two in silico algorithms predicted an effect on mRNA splicing (Online Supplementary Table S3) that was confirmed in vivo even though with a slight effect. To study c.4866C $>$ T, the exon 25-31 region was amplified and sequenced, but no effect on mRNA processing was seen, as was predicted by in silico tools. Both nucleotides were identified in heterozygous state, indicating the presence of both alleles. Only offspring carrying the c.546G $>\mathrm{A}$ mutation (1 of 3 children) had lower levels of VWF:Ag and VWF:RCo compared to 
Table 2. Effect of VWF mutations on mRNA from leukocytes and platelets.

\begin{tabular}{|c|c|c|c|c|c|c|c|}
\hline Mutation type & e NT change & AA change & Exon & Intron & Leukocyte effect & Platelet effect & Protein prediction \\
\hline \multirow[t]{9}{*}{ Intronic } & c. $1533+1 \mathrm{G}>\mathrm{A}$ & - & - & 13 & Exon 13 skipping (r.1433_1533del) & NMD & p.Gly478AlafsTer138 \\
\hline & c. $3379+1 \mathrm{G}>\mathrm{A}^{*}$ & - & - & 25 & Exon 25 skipping (r.3223_3379del) & NMD & p.Pro1075ValfsTer88 \\
\hline & & & & & $\begin{array}{l}\text { Activation of a cryptic site at }+126 \mathrm{nt} \\
\text { in exon } 25 \text { (r.3349_3379del) } \ddagger\end{array}$ & NMD & p.Prol117ValfsTer88 \\
\hline & c. $5664+2 \mathrm{~T}>\mathrm{C}$ & - & - & 33 & Exon 33 skipping (r.5621_5664del) & NMD & p.Gly1874AlafsTer32 \\
\hline & & & & & Exon 33+34 skipping (r.5621_5842del) $\$$ & NMD & p.Phe1875_Cys1948del \\
\hline & c.7082-2A>G $\dagger$ & - & - & 41 & $\begin{array}{l}\text { Activation of a cryptic site }+7 \mathrm{nt} \\
\text { in exon } 42 \text { (r.7082_7088del) }\end{array}$ & NMD & p.Ala2361GlyfsTer40 \\
\hline & c. $7730-4 \mathrm{C}>\mathrm{G}$ & - & - & 45 & No visible effect & No visible effect & - \\
\hline & c. $7730-56 \mathrm{C}>\mathrm{T}$ & - & - & 45 & No visible effect & No visible effect & - \\
\hline & c. $8254-5 \mathrm{~T}>\mathrm{G}$ & - & - & 51 & No visible effect & No visible effect & - \\
\hline & c. $7437 \mathrm{G}>\mathrm{A}$ & p.Ser2479 & 43 & - & Exon 43 skipping (r.7288_7437) & NA & p.Val2430GlyfsTer335 \\
\hline & & & & & $\begin{array}{l}\text { Activation of a cryptic site at }+146 \mathrm{nt} \\
\text { in exon } 43 \text { (r.7434_7437del) }\end{array}$ & NA & p.Ser2479AlafsTer23 \\
\hline \multirow[t]{3}{*}{ Missense } & c. $449 \mathrm{~T}>\mathrm{C}$ & p.Leul50Pro & 5 & - & No visible effect & No visible effect & - \\
\hline & c. $1109 \mathrm{G}>\mathrm{A}$ & p.Cys370Tyr & 9 & - & Exon 9 skipping (r.998_1109del) & NMD & p.Glu333AlafsTer87 \\
\hline & & & & & Exon 8+9 skipping (r.875_1109del) & NMD & p.Ser292ThrfsTer87 \\
\hline \multirow[t]{3}{*}{ DelIns } & 485_3486delinsTG & p.Proll62Leu & 26 & - & No visible effect & No visible effect & - \\
\hline & .3223-7_3236dup p. & Pro1079_Tyr1080insLe & & & & & \\
\hline & & GlnValAspProGluPro & 25 & - & (r.3477_3478instttgcaggtggaccccgagcc) & $\begin{array}{l}\text { (r.3477_3478instttgcaggt } \\
\text { ggaccccgagcc) }\end{array}$ & $\begin{array}{l}\text { p.Pro1079_Tyr1080insLeu } \\
\text { GlnValAspProGluPro }\end{array}$ \\
\hline
\end{tabular}

AA:amino acid; NA: not available; NMD: nonsense-mediated decay; NT:nucleotide. *Previous functional studies by Nesbitt et al. ${ }^{23}$ Previous functional studies by Corrales et al. ${ }^{7}$ Leukocyte effect observed in a really low $\%$ of reads by NGS.

the others. This may be explained by the mutation and/or other factors not explored in the current study.

The c.3291C>T mutation (exon 25), was identified in patient UMP09 (female), as well as a novel mutation in F8 (c.1346C > G, p.Ala430Gly). Of note, her brother, who was not included in this study, also had these mutations. As both siblings experienced bleeding, and the HSF prediction regarding c.3291C $>\mathrm{T}$ interpreted creation of an exonic splicing silencer site that could potentially alter splicing, we hypothesized that this synonymous mutation could have an effect on VWF mRNA processing. Hence, the exon 22-26 region was amplified, but no change in the $V W F$ cDNA sequence was observed. In addition, we tested whether intron 25 was retained within mature VWF mRNA (Online Supplementary Methods), but no differences compared to control leukocyte cDNA were found (data not shown). Finally, as both siblings presented similar FVIII:C levels, we investigated $\mathrm{X}$ chromosome inactivation in patient UMP09. The results demonstrated skewed inactivation of the WT X chromosome in this patient (Online Supplementary Figure S5).

\section{Missense mutations}

The mutation c.1109G>A (p.Cys370Tyr, exon 9) was identified in a type 3 VWD carrier (UMP10). The exon 6-
12 region was investigated. Whereas the expected size was observed in platelets, amplification from leukocytes resulted in 2 additional bands of $\sim 650$ and $~ 500$ bp (Figure $3)$. Sanger sequencing of leukocyte mRNA confirmed that the 650-bp band corresponded to a transcript lacking exon 9. NGS of leukocyte mRNA revealed 3 different transcripts: the WT, a transcript skipping exon 9 (p.Glu333AlafsTer87) (13\% of reads), and a transcript skipping exons 8 and 9 (p.Ser292ThrfsTer87) (7\% of reads)(Table 2 ). Sequencing of PCR products from platelets showed no changes, suggesting that the mutated allele had experienced NMD.

\section{Duplication mutation}

The c.3223-7_3236dup mutation, which generated an in-frame tandem duplication of 21 nucleotides, including 7 nucleotides from intron 24 and the first 14 from exon 25, was detected in a type 1 VWD patient (UMP11). Study of this mutation by the two techniques demonstrated a change in the native ASS of intron 24 in both cell types, which resulted in maintaining the 21-bp insert corresponding to 7 aberrant amino acids in mature VWF mRNA (p.Pro1079_Tyr1080insLeuGlnValAspProGluPro) (Table 2 and Online Supplementary Figure S6). Furthermore, NGS showed that the aberrant mRNA transcript was 
present in $\sim 14 \%$ of reads in both cell types (Online Supplementary Table S4).

\section{Combined potential splice site mutations in individual patients}

The c.7082-2A > G mutation (intron 41) was identified in a type 3 VWD patient (UMP12) combined in trans with the p.Leu150Pro (c.449C>T, exon 5) mutation. To study c.7082-2A>G, the exon 38-43 region was amplified in platelets and leukocytes, which yielded a band of the expected size. However, Sanger and NGS sequencing of the PCR products revealed activation of a cryptic ASS within exon 42. This led to deletion of the 7 initial nucleotides of exon 42 (r.7082_7088del), as was predicted by the 4 algorithms, and resulted in a frameshift and a PTC (p.Ala2361Glyfs Ter40) (Figure 4). Additionally, NGS showed that the aberrant transcript was present in $24 \%$ of reads. On sequencing of platelet amplicons, there were no changes, a finding highly suggestive of NMD. As type 3 VWD is characterized by absent or non-functional expression of both VWF alleles, we postulated that the p.Leu150Pro missense mutation could have an effect on VWF mRNA. Nonetheless, no sequence change was identified. These results were confirmed by analysis of 2
A

Exon 41

Exon 42

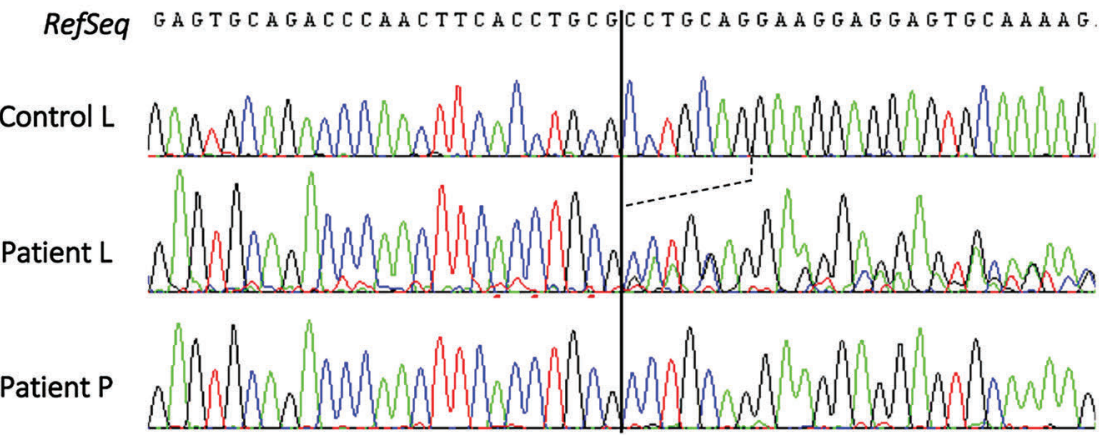

B

Exons mRNA VWF
Output BVD analysis

C

DNA

RNA - Cryptic splice site activation

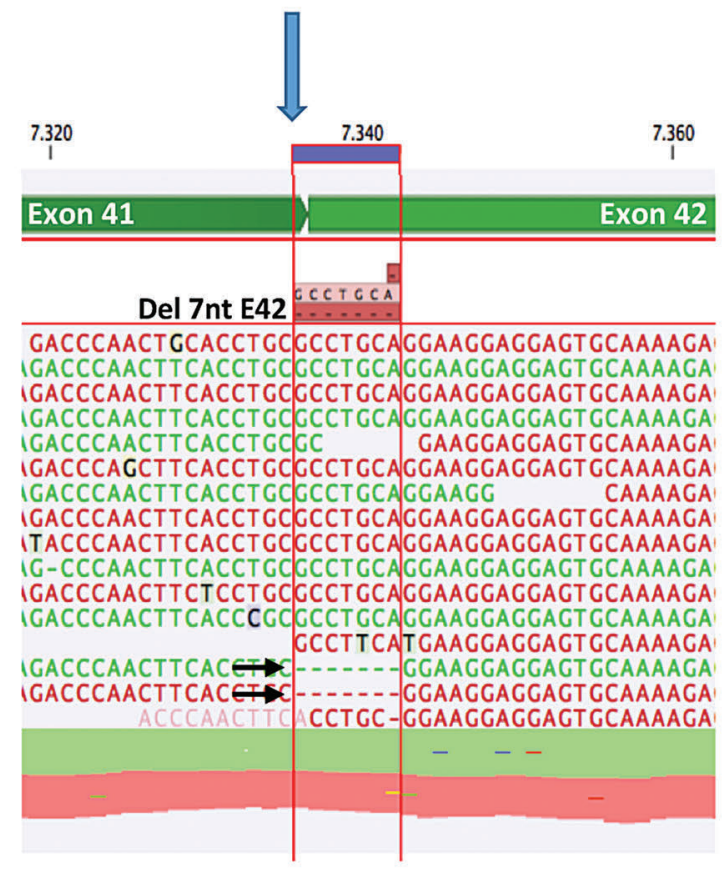

Coverage
Figure 4. Analysis of the c.7082-2A>G mutation in patient UMP12. Agarose gel electrophoresis results of RTPCR amplification of exon 38 to 43 using RNA from leukocytes and platelets were the same as those of healthy controls (data not shown). A) Traditional Sanger sequencing of PCR product from patient leukocyte (L) demonstrate activation of a cryptic splice site 7 nucleotides downstream of the native splice site within exon 42 . In patient platelet $(P)$, only the allele carrying the p.Leu150Pro mutation could be amplified. B) NGS of PCR products from leukocytes showed deletion of the 7 initial nucleotides of exon 42 Arrows show aberrant transcripts. C) Schematic representation of the mutation in genomic DNA and its effect on the VWF mRNA sequence. WO: without. 
informative SNPs, rs1800375 and rs1800376, which were found in heterozygous state in leukocyte transcripts and homozygous state in platelets.

The c.[3426T>C; 3485_3486delins TG] mutations (exon 26) were found in a type $2 \mathrm{~A}$ VWD patient (UMP13), combined in trans with p.Cys2773Ser. In silico analysis predicted no impact on the splice site for c. 3485_3486delinsTG, but 2 of the 4 algorithms predicted that c.3426T>C would have an effect on splicing. The exon 25-28 region was investigated, but no effect was found on mRNA processing. Study of the p.Cys2773Ser mutation and 2 informative SNPs (rs2228317 and rs4021576) confirmed that the 2 alleles were present, suggesting that exon 26 mutations do not have an effect on VWF splicing.

The mutations (c.7437G>A exon 43), and c.6699_6702dup (p.Cys2235ArgfsTer8, exon 38) were identified in trans in a severe type 1 VWD patient (UMP14) and studied only in leukocyte RNA. For c.7437G >A, we amplified exons $41-45$, and 2 bands emerged: one with the expected size, and a smaller band of $\sim 350$ bp (Figure 5). Sequencing analysis by both tech- niques revealed 2 aberrant transcripts: one lacked exon 43 (p.Val2430GlyfsTer335) and the other showed activation of a cryptic DSS within exon 43, leading to deletion of its 4 final nucleotides (p.Ser2479AlafsTer23) (Table 2). Study of c.6699_6702dup in leukocyte mRNA showed no splicing changes, but the duplication introduced 4 nucleotides that changed the reading frame and generated a PTC (p.Cys2235ArgfsTer8).

The c.546G>A (p.=, exon 6) and c.7082-2A>G (intron 41) mutations in trans with c. $8155+3 \mathrm{G}>\mathrm{C}$ (intron 50) were detected in a type 3 VWD patient (UMP15). This patient had been described in our previous article, but at that time we were only able to identify the effect of c. $8155+3 \mathrm{G}>\mathrm{C}$ (p.Gly2706ValfsTer24) on splicing. On reanalysis of this case with NGS, we were able to characterize the effect of c.546G >A (p.Thr179ProfsTer31) and c.7082-2A>G (p.Ala2361GlyfsTer40) in cis in leukocyte mRNA. The results are consistent with those observed in patients UMP08 and UMP12, who harbored each mutation individually. In addition, in leukocytes, transcripts resulting from c.546G $>$ A were detected in $2 \%$ of reads, transcripts
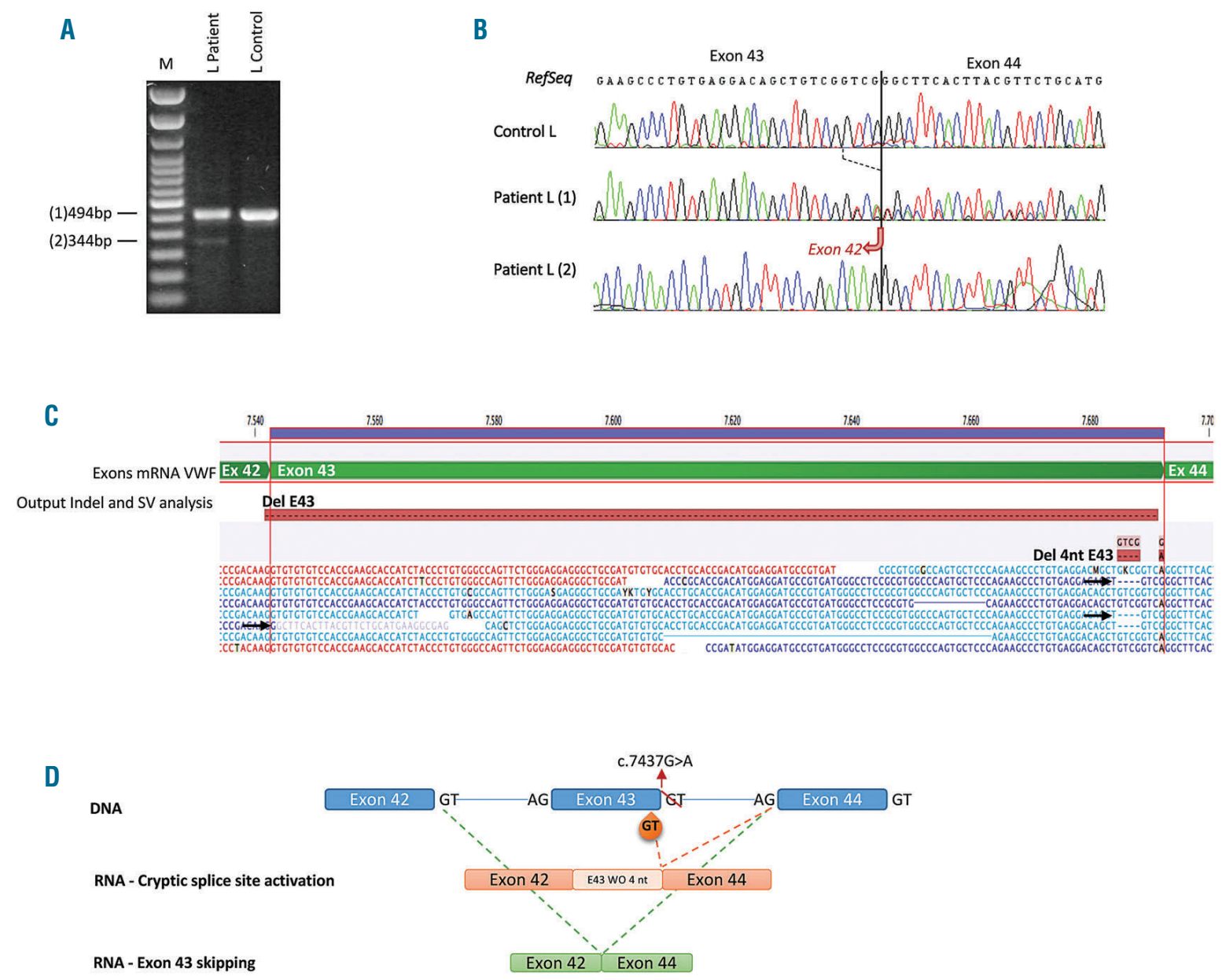

Figure 5. Analysis of the c.7437G>A ( $\mathbf{p}=$ ) mutation in patient UMP14, located in the last nucleotide of exon 43. A) RT-PCR products amplified with primers located in exons 41 and 45 in leukocyte RNA (L), separated on 1\% agarose gel. B) Traditional Sanger sequencing of PCR product from patient leukocyte (L) shows two aberrant transcripts: activation of a cryptic splice site - 4 nucleotides upstream to WT-DSS - in exon 43 (1), and exon 43 skipping (2). C) NGS of PCR products gave the same results than Sanger sequencing. D) Schematic representation of the mutation in genomic DNA and its effect on the VWF mRNA sequence. M indicates a 100bp DNA ladder. WO: without. 
from c.7082-2A>G in $6 \%$ and transcripts from c. $8155+3 G>C$ in $95 \%$ of reads. In platelet analyses, NGS detected transcripts without exon 6 resulting from the c. $546 \mathrm{G}>\mathrm{A}$ mutation in $11 \%$ of the total reads but no aberrant transcript derived from the mutation c.7082-2A $>\mathrm{G}$ indicating that NMD rate could differ between $5^{\prime}$ and $3^{\prime}$ regions of mRNA. These results indicate the c. [546G $>A$; 7082-2A>G] allele is under-represented compared to the allele carrying the $c .8155+3 \mathrm{G}>\mathrm{C}$ mutation, showing that this allele underwent NMD in both cell types, as was previously hypothesized.'

\section{Discussion}

This study reports the results of in-depth analysis of 18 PSSM in samples from VWD patients. The novel and robust procedure used, combining two-step RT-PCR and NGS sequencing, was faster and more sensitive than the method used in our previous article.? This new approach provides several advantages, such as allele-specific individual sequences, higher sensitivity to detect transcript variants present at low copy numbers, and simplified sample preparation. Moreover, the NGS data on total reads obtained for each transcript is additional information that can provide an approximation of the expression levels of each VWF mRNA. Studies are currently ongoing to confirm that the relative expression of each transcript obtained by NGS is comparable to that provided by realtime RT-PCR.

The proven usefulness of leukocyte analysis to interpret mutations masked by NMD in platelets was seen in relation to the c.3379+1G>A mutation, which had been previously investigated by RT-PCR in platelets. ${ }^{23}$ In that study, the true pathogenic effect of this mutation could not be determined due to $\mathrm{NMD}$. In the present study, using leukocyte mRNA and NGS technology, we found that the mutation induces two aberrant transcripts.

Similarly, the pathogenic mechanism of 7 PSSM (c. $1533+1 \mathrm{G}>\mathrm{A}$, c. $5664+2 \mathrm{~T}>\mathrm{C}$, c.7082-2A>G, c.546G>A, c.7437G $>\mathrm{A}$, and p.Cys370Tyr) was determined in leukocytes, and only the c.3223-7_3236dup mutation effect was observed in both cell types. Because this mutation is located in the D3 domain implicated in multimerization, it is possible that the pathogenic mechanism leading to type 1 VWD may be related to impaired VWF secretion due to intracellular retention, as has been described for other D3 mutations such as p.Cys1130Phe. ${ }^{24}$ To confirm this hypothesis, expression studies by means of heterologous cell lines or blood outgrowth endothelial cells (BOECs) will remain the gold standard.

As would be expected, mutations located in the consensus splicing sequence affected mRNA processing. These included c. $1533+1 \mathrm{G}>\mathrm{A}$, c.5664+2T>C, leading to exon skipping, and c.7082-2A>G, activating a cryptic splice site. Both these molecular effects have been reported previously in splicing mutations causing VWD. ${ }^{7,25,26}$ The c.7082$2 \mathrm{~A}>\mathrm{G}$ in trans with p.Leu150Pro leads to the development of type 3 VWD. The p.Leu150Pro mutation does not affect splicing. However, other mutations located in the propeptide, such as p.Asp141Tyr, have been identified in type 3 patients, ${ }^{27}$ and, as has been described in these cases, we suspect that p.Leu150Pro may compromise propeptide folding and affect intracellular survival and the capacity to mediate multimerization. The c.1533+1G>A mutation in leukocytes generated three aberrant transcripts. One of them, which leads to the inframe deletion of exons 13-14, should have been detected in platelets since it would not generate a PTC and would not be degraded. However, because the mutation is located in a region with weak splicing signal sequences, as previously demonstrated, ${ }^{7,25,26}$ we suggest that the results obtained in leukocytes could be an artifact that would not occur in the natural cellular type of VWF expression. Based on these observations, we propose that the real effect of c.1533+1G>A is the skipping of exon 13 , which predicts a frameshift at position 478 and addition of 138 aberrant amino acids before a PTC is encountered (p.Gly478AlafsTer138).

Two synonymous mutations included in our study, c.546G $>A$ and c.7437G $>A$, affect splicing of VWF mRNA. To our knowledge, only three synonymous mutations affecting VWF mRNA processing have been reported: c. $7056 \mathrm{C}>\mathrm{T},{ }^{28}$ c. $7464 \mathrm{C}>\mathrm{T},{ }^{29}$ and c. $3390 \mathrm{C}>\mathrm{T} .{ }^{26}$ Of note, the results of the present study have almost doubled the number of reported mutations of this type causing VWD. The pathogenic effect of c.546G $>$ A could only be documented by NGS; it eluded Sanger detection because of the low expression in leukocytes and platelets. The c.7437G $>$ A mutation located at the last nucleotide of exon 43 was found in a patient with severe type 1 VWD (UMP14) in trans with c.6699_6702dup (p.Cys2235ArgfsTer8). This synonymous mutation produced 2 splicing variants. First, creation of a new DSS 4 nucleotides upstream of the WTDSS in exon 43 that generated a PTC in exon 44 and would lead to NMD in platelets; and second, exon 43 skipping and generation of a PTC in exon 52. In this latter case, we would expect that NMD had been abolished in platelets, since this cellular mechanism is not effective when a PTC is encountered within $50 \mathrm{bp}$ of the last exonexon junction. ${ }^{30}$

Study of the p.Cys370Tyr (c.1109G $>$ A in exon 9) mutation, resulted in generation of 2 VWF transcripts, one of them identified only by NGS because of their low expression. Of note, only one other missense mutation, p.Gly1108Arg, has been reported to affect VWF splicing. Thus, these two mutations support the concept that missense mutations on the last exonic nucleotides can also have repercussions on the splicing process. ${ }^{31}$ Interestingly, the pathogenic effect of this mutation is the same as that described for c. $1109+2 \mathrm{~T}>\mathrm{C}$ (intron 9) ${ }^{32}$ In addition to the patient reported here, the p.Cys370Tyr mutation was identified in 6 additional related patients included in the PCM-EVW-ES: in homozygous state in a type 3 VWD patient and in heterozygosis in 4 type 3 carriers and 1 patient with type 1 VWD, based on their phenotype levels. Certain genetic modifiers of VWF levels ${ }^{33}$ and interindividual variability in NMD efficiency between patients carrying identical mutations may lead to differences in the disease severity and clinical phenotype. ${ }^{34}$

Lastly, study of c.7081+6G $>$ T, c.7730-4C>G, c.7730$56 \mathrm{C}>\mathrm{T}, \quad$ c. $8254-5 \mathrm{~T}>\mathrm{G}, \quad$ c. $3291 \mathrm{C}>\mathrm{T}, \quad$ c. $4866 \mathrm{C}>\mathrm{T}$ and c.[3426T>C; 3485_3486delinsTG] showed no visible effect on mRNA processing, although this does not necessarily mean that they have no effect on splicing. For instance, mRNA SNP analysis in patient UMP05 with c.7730-56C $>\mathrm{T}$ showed an absence of 1 allele in leukocytes and platelets, suggesting that the allele may have experienced NMD in both cell types or lack expression due to a mutation that was not detected by our sequencing protocol. Of particular note, deep intronic mutations such as 
c.6599-20A > T have been found to cause VWD. ${ }^{35}$ In patient UMP07 carrying the c.8254-5T>G mutation, no informative SNP was identified and we were unable to determine whether there was a lack of expression of 1 allele, which could explain cosegregation of the mutation in the family with bleeding symptoms.

Study of c.[3426T>C; 3485_3486delinsTG] and c.7730$4 \mathrm{C}>\mathrm{G}$ showed no visible effect on splicing. However, these mutations have been identified in homozygous state in a type 3 patient (c.[3426T>C; 3485_3486delins TG]) ${ }^{3}$ and in a severe type 1 VWD patient $(c .7 \overline{7} 30-4 C>G)^{4}$ with VWF:Ag at 7\% and VWF:RCo at 5\%, combined with the heterozygous p.Ala631Val (previously reported in a healthy control). ${ }^{36}$ Based on these findings, there is substantial evidence that these mutations would have an effect on VWF levels. Therefore, to unequivocally determine the potential deleterious effect of these variants, functional studies remain essential. These studies are traditionally carried out by in vitro analyses performed using heterologous cell lines (COS7, AtT-20 and HEK293). However, the advent of the possibility of obtaining BOECs from patients represents a valuable alternative, since this is the functional expression site of $V W F .^{37}$ Moreover, BOECs allows protein expression and mRNA studies simultaneously.

In silico algorithms used to assess the impact of mutations on splicing are more sensitive and accurate in determining the putative effect of intronic mutations than that of syn- onymous or missense mutations, such as c.546G $>A$ and p.Cys370Tyr. Therefore, the results here should not be considered definitive, and as with all analytical approaches, should form one aspect of a wider investigation. ${ }^{5}$

In conclusion, we present an extensive study reporting the effect of 18 candidate mutations on VWF mRNA processing. In vivo mRNA studies incorporating NGS technology together with traditional sequencing enabled us to determine the pathogenic effect of 8 PSSM (44\%). Our study emphasizes the importance of examining selected mutations, including synonymous and missense mutations, to determine their pathogenic role in splicing. Taken together, our results add to the current knowledge about the molecular events leading to VWD.

\section{Acknowledgments}

We are indebted to Baxalta US Inc., now a part of Shire, for support of the PCM-EVW-ES (Grant H13-000845).

\section{Funding}

This study was also supported by the Spanish Ministry of the Economy and Competitiveness (MINECO, Ministerio de Economia y Competitividad), Instituto de Salud Carlos III (ISCIII) (PI12/01494, PI15/01643 and RD12/0042/0053). We are very grateful for the kind collaboration of the participating patients and their families. CIBERCV is an initiative of ISCIII, co-financed by the European Regional Development Fund $(E R D F)$, "A way to build Europe".

\section{References}

1. Rodeghiero F, Castaman G, Dini E. Epidemiological investigation of the prevalence of von Willebrand's disease. Blood. 1987;69(2):454-459

2. Batlle J, Perez-Rodriguez A, Corrales I, et al. Molecular and clinical profile of von Willebrand disease in Spain (PCM-EVWES): Proposal for a new diagnostic paradigm. Thromb Haemost. 2016;115(1):4050 .

3. Borras N, Batlle J, Perez-Rodriguez A, et al. Molecular and clinical profile of von Willebrand disease in Spain (PCM-EVWES): comprehensive genetic analysis by next-generation sequencing of 480 patients. Haematologica. 2017;102(12): 2005-2014.

4. Fidalgo T, Salvado R, Corrales I, et al. Genotype-phenotype correlation in a cohort of Portuguese patients comprising the entire spectrum of VWD types: impact of NGS. Thromb Haemost. 2016;116(1):17-31.

5. Richards S, Aziz N, Bale S, et al. Standards and guidelines for the interpretation of sequence variants: a joint consensus recommendation of the American College of Medical Genetics and Genomics and the Association for Molecular Pathology. Genet Med. 2015;17(5):405-424.

6. Wang QY, Song J, Gibbs RA, Boerwinkle E, Dong JF, Yu FL. Characterizing polymorphisms and allelic diversity of von Willebrand factor gene in the 1000 Genomes. J Thromb Haemost. 2013;11(2):261-269.
7. Corrales I, Ramirez L, Altisent C, Parra R, Vidal F. The study of the effect of splicing mutations in von Willebrand factor using RNA isolated from patients' platelets and leukocytes. J Thromb Haemost. 2011;9(4):679-688.

8. Wang GS, Cooper TA. Splicing in disease: disruption of the splicing code and the decoding machinery. Nat $\mathrm{Re}$ Genet. 2007;8(10):749-761.

9. Eikenboom JC, Reitsma PH, Peerlinck KM Briët E. Recessive inheritance of von Willebrand's disease type I. Lancet. 1993;341(8851):982-986.

10. Jaffe EA, Hoyer LW, Nachman RL Synthesis of von Willebrand factor by cultured human endothelial cells. Proc Natl Acad Sci U S A. 1974;71(5):1906-1909.

11. Sporn LA, Chavin SI, Marder VJ, Wagner DD. Biosynthesis of von Willebrand protein by human megakaryocytes. J Clin Invest. 1985;76(3):1102-1106.

12. Kieffer N, Guichard J, Farcet JP, Vainchenker W, Breton-Gorius J. Biosynthesis of major platelet proteins in human blood platelets. Eur J Biochem. 1987;164(1):189-195.

13. Fink L, Holschermann H, Kwapiszewska $\mathrm{G}$, et al. Characterization of platelet-specific mRNA by real-time PCR after laserassisted microdissection. Thromb Haemost. 2003;90(4):749-756.

14. McVey JH, Boswell EJ, Takamiya $O$, et al. Exclusion of the first EGF domain of factor VII by a splice site mutation causes lethal factor VII deficiency. Blood. 1998;92(3):920-926.

15. David D, Santos IM, Johnson K,
Tuddenham EG, McVey JH. Analysis of the consequences of premature termination codons within factor VIII coding sequences. J Thromb Haemost. 2003;1(1): 139-146.

16. Roberts RG, Bentley DR, Bobrow M. Infidelity in the structure of ectopic transcripts: a novel exon in lymphocyte dystrophin transcripts. Hum Mutat. 1993;2 (4):293-299.

17. Byers PH. Killing the messenger: new insights into nonsense-mediated mRNA decay. J Clin Invest. 2002;109(1):3-6.

18. Lewis BP, Green RE, Brenner SE. Evidence for the widespread coupling of alternative splicing and nonsense-mediated mRNA decay in humans. Proc Natl Acad Sci U S A. 2003;100(1):189-192.

19. Martorell L, Luce E, Vazquez JL, et al. Advanced cell-based modeling of the royal disease: characterization of the mutated F9 mRNA. J Thromb Haemost. 2017;15(11): 2188-2197.

20. Hojny J, Zemankova P, Lhota F, et al. Multiplex PCR and NGS-based identification of mRNA splicing variants: Analysis of BRCA1 splicing pattern as a model. Gene. 2017;637:41-49

21. Brunak S, Engelbrecht J, Knudsen S Prediction of human mRNA donor and acceptor sites from the DNA sequence. J Mol Biol. 1991;220(1):49-65.

22. Corrales I, Ramirez L, Altisent C, Parra R, Vidal F. Rapid molecular diagnosis of von Willebrand disease by direct sequencing. Detection of 12 novel putative mutations in VWF gene. Thromb Haemost. 2009;101(3):570-576. 
23. Nesbitt IM, Hampton KK, Preston FE, Peake IR, Goodeve AC. A common splice site mutation is shared by two families with different type $2 \mathrm{~N}$ von Willebrand disease mutations. Thromb Haemost. 1999;82(3):1061-1064.

24. Tjernberg P, Vos HL, Castaman G, Bertina RM, Eikenboom JC. Dimerization and multimerization defects of von Willebrand factor due to mutated cysteine residues. J Thromb Haemost. 2004;2(2):257-265.

25. Gallinaro L, Sartorello F, Pontara E, et al. Combined partial exon skipping and cryptic splice site activation as a new molecular mechanism for recessive type 1 von Willebrand disease. Thromb Haemost. 2006;96(6):711-716.

26. Pagliari MT, Baronciani L, Garcia Oya I, et al. A synonymous (c.3390C > T) or a splicesite (c.3380-2A>G) mutation causes exon 26 skipping in four patients with von Willebrand disease (2A/IIE). J Thromb Haemost. 2013;11(7):1251-1259.

27. Baronciani L, Federici AB, Cozzi G, et al. Expression studies of missense mutations p.D141Y, p.C275S located in the propeptide of von Willebrand factor in patients with type 3 von Willebrand disease Haemophilia. 2008;14(3):549-555.

28. Daidone V, Gallinaro L, Grazia Cattini M et al. An apparently silent nucleotide substitution (c.7056C $>\mathrm{T}$ ) in the von Willebrand factor gene is responsible for type 1 von Willebrand disease. Haematologica. 2011;96(6):881-887.

29. Yadegari H, Biswas A, Akhter MS, et al. Intron retention resulting from a silent mutation in the VWF gene that structurally influences the $5^{\prime}$ splice site. Blood. 2016;128(17):2144-2152.

30. Maquat LE. Nonsense-mediated mRNA decay: splicing, translation and mRNP dynamics. Nat Rev Mol Cell Biol. 2004;5(2): 89-99.

31. James PD, O'Brien LA, Hegadorn CA, et al A novel type $2 \mathrm{~A}$ von Willebrand factor mutation located at the last nucleotide of exon 26 (3538G $>A$ ) causes skipping of 2 nonadjacent exons. Blood. 2004;104(9): 2739-2745.

32. Castaman G, Plate M, Giacomelli SH, Rodeghiero F, Duga S. Alterations of mRNA processing and stability as a pathogenic mechanism in von Willebrand factor quantitative deficiencies. I Thromb Haemost. 2010;8(12):2736-2742.

33. James PD, Lillicrap D. von Willebrand disease: clinical and laboratory lessons learned from the large von Willebrand disease studies. Am J Hematol. 2012;87 Suppl 1:S4-11.

34. Miller JN, Pearce DA. Nonsense-mediated decay in genetic disease: friend or foe? Muta Res Rev Mutat Res. 2014;762:52-64.

35. Hawke L, Bowman ML, Poon MC, Scully MF, Rivard GE, James PD. Characterization of aberrant splicing of von Willebrand factor in von Willebrand disease: an underrecognized mechanism. Blood. 2016;128(4) 584-593.

36. Bellissimo DB, Christopherson PA, Flood $\mathrm{VH}$, et al. VWF mutations and new sequence variations identified in healthy controls are more frequent in the AfricanAmerican population. Blood. 2012;119(9): 2135-2140.

37. Wang JW, Bouwens EA, Pintao MC, et al Analysis of the storage and secretion of von Willebrand factor in blood outgrowth endothelial cells derived from patients with von Willebrand disease. Blood. 2013;121 (14):2762-2772 